\title{
COGNITIVE RADIO AND RADIO FREQUENCY IDENTIFICATION TECHNOLOGIES IN SMART ENVIRONMENT
}

\author{
Roa Alharbi \\ Department of Computer Engineering, \\ University of Western Ontario, London, Canada
}

\begin{abstract}
In this survey, smart environment wireless networks are reviewed. The focus is on using the Cognitive Radio (CR) technology and Radio Frequency Identification (RFID) technology in these smart networks. Therefore, the reviewed research papers are studying the Cognitive Radio usage in smart homes and hospitals wireless networks. The main objectives of using CR in wireless networks are providing the flexibility in networks allocation, managing wireless networks efficiently and saving time and cost for communications. In addition, the papers include how the Radio Frequency Identification technology is merged with the Cognitive Radio to produce a smart environment. Although new technologies like CR and RFID have advantages, they also have disadvantages such as network interferences, network allocation and users' priorities management. For this reason, recent studies are describing the challenges that facing CR and RFID technologies and new ways to solve them.
\end{abstract}

\section{KEYWORDS}

SDR, Cognitive Radio

\section{INTRODUCTION}

The smart environment is a small world that includes all person's requirements in a fast and comfortable way. This small world has smart devices that are continuously working to get those requirements anytime and anywhere. There are three different types of smart environment: virtual computing environment, physical environment and human environment. Initially, virtual computing environment is a virtual world which allows smart devices to access specific services. Secondly, physical environment includes various types of smart devices such as tags, controllers and sensors. Thirdly, human environment where the human is included in this technology, for example using mobile phones and other devices.

Focusing on physical environment, there are several technologies to build this kind of environment. Software Defined Radio (SDR) technology is one of the technologies. To explain, SDR is defined by the Institute of Electrical and Electronic Engineers (IEEE) as a "Radio in which some or all of the physical layer functions are software defined"[1]. So that, SDR makes Natarajan Meghanathan et al. (Eds) : NeTCoM, CSEIT, GRAPH-HOC, NCS, SIPR - 2017 pp. 37- 44, 2017. @ CS \& IT-CSCP 2017

DOI : $10.5121 /$ csit.2017.71504 
the communication more flexible by modifying radio devices. Moreover, using Software Defined Radio technology imports the cost efficiency in the communication business area. In general, any device that uses radio frequency (FR) spectrum to transfer and receive signals in a wireless network is called a "radio". Radio devices are used today as cellphones, televisions and computers. Further, SDR technology leads to three related technologies which are Adaptive Radio, Cognitive Radio and Intelligent Radio. Adaptive Radio technology enables communication systems to control their performance and edit their operating features like frequency, data and power. However, communication systems that use Cognitive Radio know their location in RF spectrum and they are designed to decide the best wireless channel and change their internal state. Intelligent Radios are cognitive radios but they use machine learning technology to improve their performance. [2]

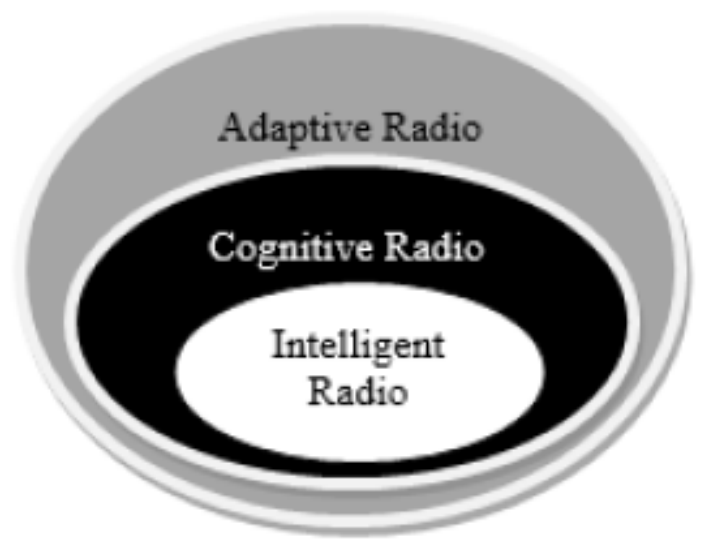

Figure 1.SDR Related Technologies [1]

Another related technology to the SDR is the Radio Frequency Identification (RFID). RFID is a wireless communication technology which enables the identification of objects with specific tags [8]. The RFID system contains of tags (electronic labels) on objects, reader (integrator) and information stored in the system's database. Each tagged object has a memory chip that saves object's identifiers such as the unique Electronic Product Code (EPC), time stamp, read count, radio frequency and received signal strength indicator [9]. In addition, the reader is connected with an antenna emits signals to the tag on the object, which distributes this signal with its ECP. In detail, tags could be active, passive or battery-assisted passive. These three kinds differ in their batteries, the active tag has its own on-board battery. The battery-assisted passive has a small on board battery that activated when it is connected with reader. However, the passive tag has no battery and it has to use radio energy to work. Further, tags could be read-only tag that are programmed from the factory and could not be changed. They could be read/write tags, which enable the end user to program specific function on these tags. RFID tags are composed of two main parts, the integrated circuit and antenna. The integrated circuit is used to process information and the antenna is used to receive and transfer signals. Similarly, readers depending on associated tags have three different types: Passive Reader Active Tag (PRAT), Active Reader Passive Tag (ARPT) and Active Reader Active Tag (ARAT). In PRAT system, the passive reader receives signals from the active tag, which transmits signals only. However, the active reader in the APRT system transmits signals and receives information from passive tags. The ARAT system has active readers activated with active tags to send and receive information [8]. Important to realize, RFID frequency bands is classified into different ranges. For example, smart 
cards utilize $13.56 \mathrm{MHz}(\mathrm{HF})$ frequency band and the range for that 10 centimetres to 1 meter. Also, Bluetooth standards use 2450-5800 MHz that range from 1 to 2 meters [3].

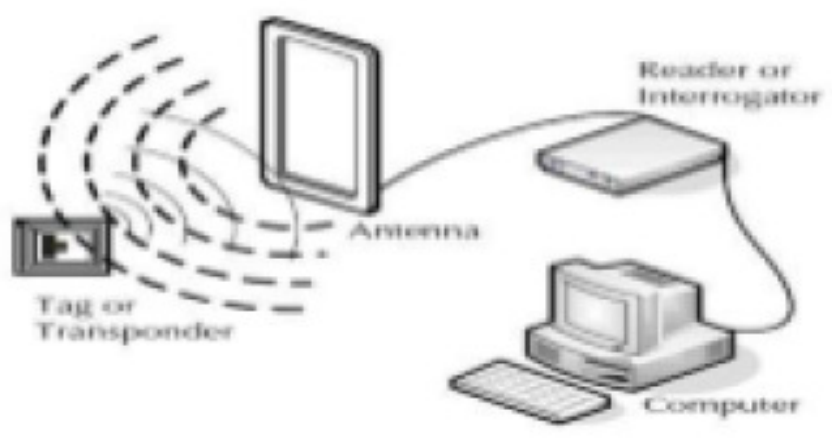

Figure 2: RFID

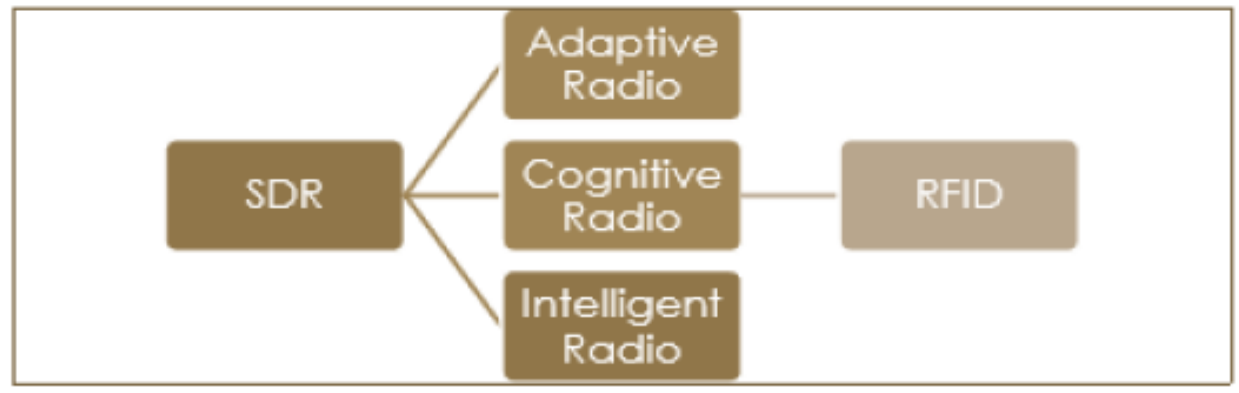

Figure 3: Survey Paper Classification

The above classification is important to understand the proposed survey paper. The SDR technology, CR technology and RFID technology are three related technologies in smart environment wireless networks. In this survey paper, I am focusing on CR study area because it is an essential technique to produce flexible wireless access, especially in large networks that are surrounding with many devices and application. This area is changing rabidly, so we as engineers must be aware of these changes and be part in introducing an improved version of these techniques.

\section{COGNITIVE RADIO TECHNOLOGY IN SMART HOMES NETWORKS}

The Using Cognitive Radio (CR) technology in building smart homes would produce better and more cost efficient environment. Cognitive Radio enables users to connect to several wireless technologies at the same time [10]. Also, users can move between these technologies easily. Further, CR technology helps in adapting to channel conditions, declining packets loses and changing in modulation. These features leads to increase energy efficiency in smart home network. Although Cognitive Radio technology provides flexibility in the management of wireless sensor networks in smart homes, it faces some challenges such as the emitted interferences and the indoor penetration losses. According to Per Lynggaard and Knud Erik Skouby in the article "Deploying 5G-Technologies in Smart City and Smart Home Wireless Sensor Networks with Interferences", they state that it has been proven in previous studies that 
the interference is a big challenge in smart homes' networks [4]. For instance, using the IEEE standard in the WIFI access point offers 14 channels in the $2.4 \mathrm{GHz}$ and each channel is $22 \mathrm{MHz}$ wide distributed by $5 \mathrm{MHz}$. In this case, an overlap is resulted. So that, CR technology is used to subdivide spectrum access intelligently to decrease overlapping. This solution has been studied by Cavalcanti, and he mentioned in his study that there is an obvious improvement in allocation channels by using CR in smart homes. To clarify, CR connects all infrastructure elements for smart home by multi-hop and pervasive techniques. These elements are able to transfer big information using band allocation which allows high bitrate transferring. As a result, emitted interferences in smart home networks could be decreased using CR technology [11]. Another key point, the indoor penetration losses challenge could be solved with the CR technology. To explain, it has been found that penetration losses depend on wall thickness and type. For example, a $12 \mathrm{~cm}$ thick uniform plaster wall would have penetration losses in the range of 5 to $10 \mathrm{~dB}$. As a solution, there are two option, one option is to increase the transmit power by reducing the Bit Error Rate (BER). The other option, is to decrease the BER by programming error correcting coding (pre-coding); however, this technique could affect the bandwidth because the pre coding generates overhead information. In the above study, the authors proved by simulations that deploying CR technology in smart home networks decrease the mentioned challenges. These simulations compares commonly used networks in smart homes with the one using CR. They found that using CR technology saves $10 \%$ power and $7 \%$ bandwidth.

Also, battery life for the smart home network could be extended to $26 \%$ with using CR in these networks [4].

\section{COGNITIVE RADIO AND RADIO FREQUENCY IDENTIFICATION IN SMART HOSPITALS}

In recent years, using wireless communication in hospitals has been a popular trend. New technologies are used in hospitals in monitoring patient's status such as blood pressure, heart been and temperature [5]. These technologies must be connected to each other to transfer patient's information through trusted wireless networks. However, wireless communication in hospitals challenges two key issues. First, the electromagnetic interferences that could influence the bi medical devices' performances. Second, the wireless channel allocation depending on devices' priorities. Speaking about these issues, there are two types of the spectrum sharing in the wireless network. The first kind, is the Horizontal Spectrum Sharing, which is accessing the unlicensed band by the radio. In this type, Cognitive Radio is used to improve the usage of the spectrum by devices in an efficient way. The other kind, is accessing the licensed band by the radio, which is called the Vertical Spectrum Sharing. In this case, Cognitive Radio is used by the secondary device (low-priority device) to access a licensed spectrum assigned to a primary device (high-priority device). Moreover, medical devices could be passive or active. Passive devices do not transmit wireless signals while Active devices transmit wireless signals and they could be interfered by other device [12].

In "A Cognitive Radio System for E-Health Applications in a Hospital Environment" paper, the authors focused on two active applications real-time non-critical telemedicine and hospital information system [6]. Telemedicine applications include remote diagnosis, consultation and patients" information transformation. While hospital information system manages patients and staffs data and stores them in hospital's database. For this study, the telemedicine is treated as the primary user and the hospital information system is treated as the secondary user of the wireless 
network. To clarify, the architecture of the Cognitive Radio System proposed in this paper includes an inventory system, a cognitive radio and cognitive radio clients. Initially, the inventory system is used to maintain hospital's devices information such as location, electromagnetic capability requirement and activity status. Theses information should be tracked using tracing system. In this case, an attached RFID tags on devices are used for the tracking system that supports the inventory system in the smart hospital. Also, RFID reader are installed among the hospital to track these tagged devices and send information to the inventory system. Secondly, the cognitive radio system uses control channel and a data channel to operate. Further, interference avoidance approach is used for the wireless access. Thirdly, cognitive radio clients, every CR client transmits its information to the CR controller. Then, the CR controller uses the information from the inventory system to control those clients. The CR controller has two interfaces, one for the control channel and the other is for the data channel. So that, it can transmit/ receive data to/from both channels at the same time. However, the CR client has one interface to transmit/receive data to/from one of the two channels (control channel, data channel) at a time. Equally important, this study focuses on improving the Electromagnetic Capability requirement, for example the device used in wireless network must limit the transmit power to decrease interferences with other devices. Moreover, the study takes in consideration the Quality of Service of the application, such as loss or delay are factors for measuring the Quality of Service. The study was performed in an area of $27 \mathrm{~m} 2$, and it is divided into nine areas, as shown in Fig. 4.

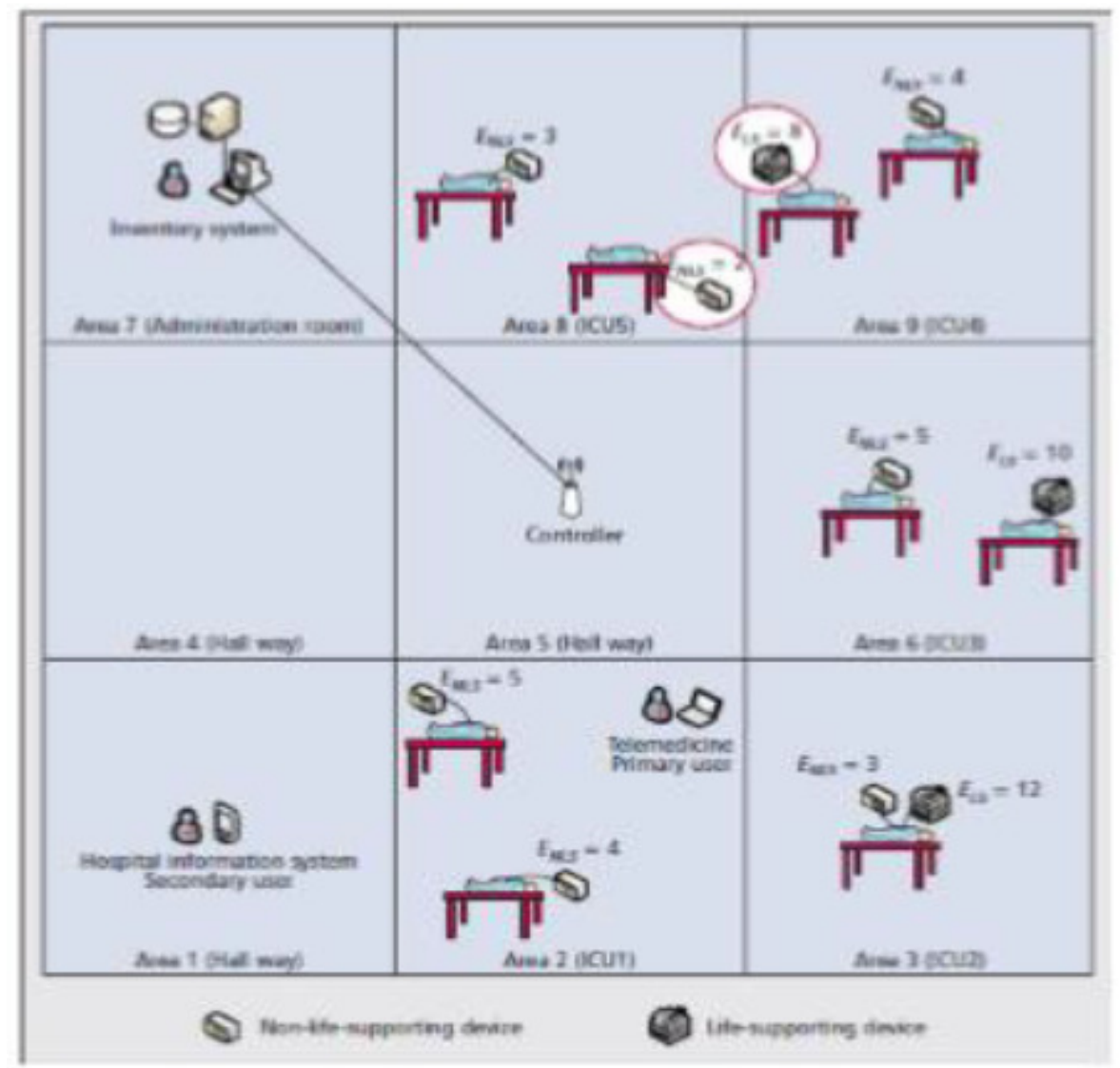

Figure 4: Cr System in Hospital [6] 
In this study, the authors include two major performance measures, the outage probability and the interference probability. The outage probability is the probability of the strength of the received signal is less than $-65 \mathrm{dBm}$ that is the minimum level for decoding in the controller. The interference probability is the probability that an interference happens, which occurs when the transmit level is higher than the acceptable level. As a result, in the CR system proposed, they found that the interference probability decreased $99.98 \%$ but the outage probability resulted is greater than the traditional wireless systems. Overall, the performance results of the proposed schema show that using CR technology in hospitals wireless networks protects the bio-medical devices from harmful electromagnetic interference. Also, the allocation performance improved by using this schema [6].

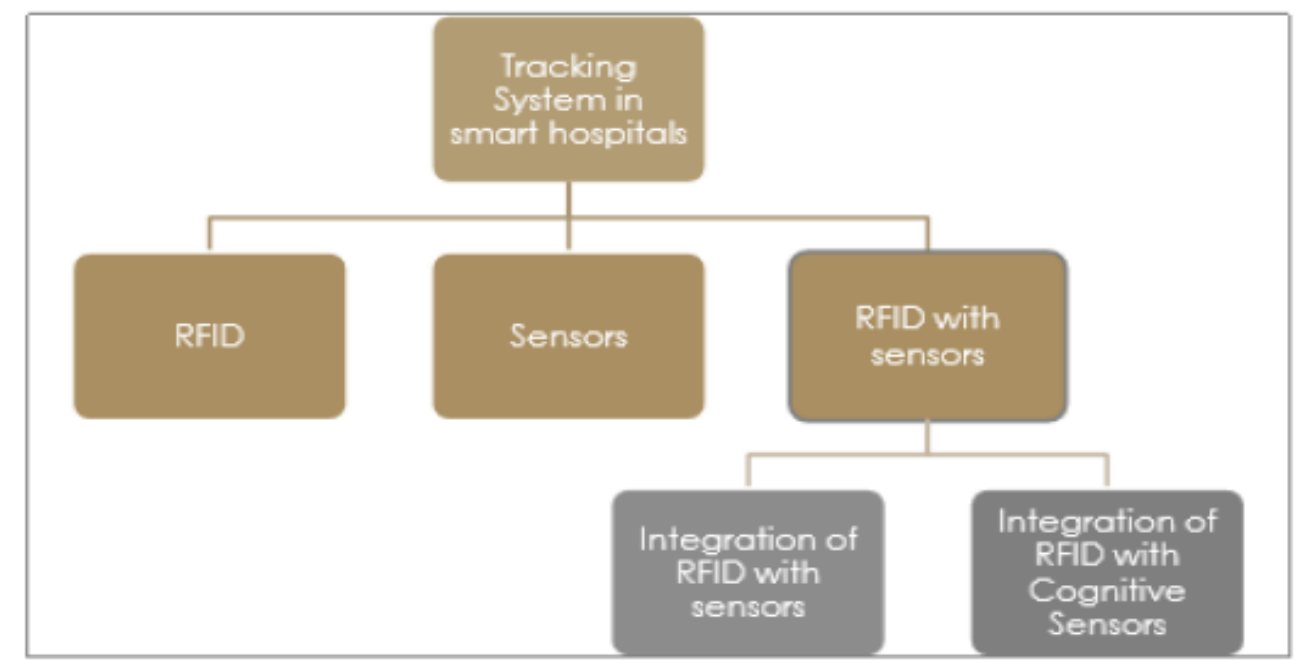

Figure 5: RFID Classification

A number of studies include integrating RFID with smart wireless networks. RFID is utilized in hospitals networks to track equipment, identify patients and improve patients care. As shown in figure 5, tracking system in smart hospitals could be done using RFID with sensors. With integrating the RFID technology with cognitive sensor, the performance of the system will be enhanced and the quality of service will be improved. Further, medical errors could be declined by using RFID. So that, each patient would be connected to his/her medicine prescription wirelessly without the need for paper prescriptions. Another important point, cloud-assisted Tracking System in smart hospitals RFID Sensors RFID with sensors Integration of RFID with sensors Integration of RFID with Cognitive Sensors integrated RFID with integrated RFID tags are useful for memory lose patients. In this technique, information is sent to doctors remotely from other places to track the patent and insure patent's safety. Also, it offers real time responses and stores patent's history with updating it each time. However, collision issue could appear with RFID technology. In this case, RFID protocol in reading tags should be developed to decrease delay as well as collision. Also, privacy could be harmed in this kind of systems. So that, restricted information security should be included. For example, a cryptography method could be used to encrypt patient's information. In this way, patient's information would be secure when transferring information among the wireless network [7]. 


\section{CONCLUSiOnS}

To sum up, a Cognitive Radio technology in smart homes wireless network is proposed, with taking in consideration, two main challenges which are the emitted interferences and the indoor penetration losses. I believe that using CR technology in communication networks adds more efficient features to these networks. Also, it saves money by reducing networks deployment costs. Moreover, the survey paper includes challenges that face smart hospitals networks. These issues are the electromagnetic interferences and the network allocation depending on users' priorities. Besides the CR technology, the RFID technology is used in these networks to track devices. As a result, there is a big improvement in networks performance with using these technologies in smart hospitals. Overall, The CR technology in smart homes and hospitals manages the network usage by categorize users as primary and secondary. This approach controls the wireless network professionally with avoiding interferences. In addition, it manages spectrums efficiently and provides users more flexibility in smart environments.

For future, Cognitive Radio system can be improved by adding improved multichannel protocols. Further, prioritization of primary and secondary devices could be controlled by stronger algorithm to gain a better Cognitive Radio system. Finally, the administration unit for the Cognitive Radio systems must control and limit number of primary users to avoid interferences in the smart environment's wireless network.

\section{REFERENCES}

[1] B. S. W. Group, "Base Station System Structure," Jan. 2010.. [Online].

[2] V. Fabrizio, "Softwrae-Defiend Radio," IEEE vehicular technology magazine, pp. 71- 82, May 2013.

[3] R. Parada , J. Melià-Seguí, M. Morenza-Cinos, A. Carreras and R. Pous, "Using RFID to Detect Interactions in Ambient Assisted Living," IEEE Computer Society, pp. 16-22, Jul. 2015.

[4] L. Per and K. E. Skouby, "Deploying 5G-Technologies in Smart City and Smart Home Wireless Sensor Networks with Interferences," Springer Science Business Media New York, Mar. 2015.

[5] Y. Pu, W. Wang and Q. Xu, "Emerging Cognitive Radio Applications: A Survey,"," IEEE Communications Magazine, pp. 74-81, Mar. 2011.

[6] P. Phunchongharn and E. Hossain, "A Cognitive Radio System for E Health Applications in a Hospital Environment," IEEE Wireless Communications, pp. 20-28, Feb. 2010.

[7] G. Prasad Joshi, S. Acharya, K. Chang-Su and K. Byung-Seo, "Smart Solutions in Elderly Care Facilities with RFID System and Its Integration with Wireless Sensor Networks," International Journal of Distributed Sensor Networks, vol. 2014, Aug 2014.

[8] K. Finkelzeller, The RFID Handbook, 2nd ed., John Wiley \& Sons, 2003.

[9] M. Weiser, "The Computer for the 21st Century," Scientific Am., vol. 265, no. 3, 1991, pp. 94-104.

[10] R. Want et al., "Bridging Real and Virtual Worlds with Electronic Tags," Proc. ACM SIGCHI, ACM Press, 1999, pp. 370-377.

[11] K. Finkelzeller, The RFID Handbook, 2nd ed., John Wiley \& Sons, 2003. 
[12] R. Want, "Enabling Ubiquitous Sensing with RFID," Computer, vol. 37, no. 4, 2004, pp. 84-86.

\section{AUTHORS}

\section{Roa Alharbi}

$>$ Researcher at KACST

$>$ Bachelor degree in Information technology, King Saud University

$>$ Master of Engineering in Software Engineering, The University of Western Ontario 